\title{
Adaptación y Validación de un Cuestionario Sobre Estrategias de Autorregulación de la Motivación en Estudiantes Universitarios
}

\section{Adaptation and Validation of a Questionnaire on Motivation Self-Regulation Strategies in College Students}

\author{
Tatiana Rojas-Ospina y Marcela Valencia-Serrano \\ Pontificia Universidad Javeriana
}

\begin{abstract}
Este estudio tuvo como objetivo evaluar las propiedades psicométricas de un cuestionario sobre estrategias de autorregulación de la motivación en jóvenes universitarios. La muestra, seleccionada intencionalmente, fue de 315 estudiantes universitarios pertenecientes a 2 universidades privadas de Cali, Colombia (edad promedio de 19 años, $51 \%$ mujeres y $49 \%$ hombres). El instrumento original en inglés (Wolters \& Benzon, 2013) fue traducido al castellano y sometido a un análisis exploratorio y confirmatorio de factores para evaluar su validez de constructo. Los resultados indican que el instrumento adaptado también presenta indicadores adecuados de validez convergente y discriminante, evaluada mediante correlaciones con el Cuestionario de Estrategias Motivacionales y de Aprendizaje (Pintrich, Smith, Garcia \& McKeachie, 1993), y de consistencia interna, para evaluar 5 de las 6 estrategias de autorregulación de la motivación consideradas en el instrumento original. El instrumento en su versión final es una herramienta útil para continuar la investigación en esta temática. Se recomienda que futuras investigaciones seleccionen muestras más grandes de estudiantes, tanto de universidades públicas como privadas, y que realicen análisis que permitan evaluar la validez predictiva del instrumento.
\end{abstract}

Palabras clave: autorregulación, motivación, universitarios, validación, consistencia interna

\begin{abstract}
The purpose of this study was to assess the psychometric properties of a questionnaire on motivation self-regulation strategies in university students. The purposive sample used comprised 315 college students from 2 private universities located in Cali, Colombia. The participants' average age was 19; 51\% were women and $49 \%$ were men. The original instrument in English (Wolters \& Benzon, 2013) was translated into Spanish and underwent exploratory and confirmatory factor analyses to evaluate its construct validity. Results indicate that the adapted instrument also displays adequate indices of convergent and discriminant validity, assessed through correlations with the Motivated Strategies for Learning Questionnaire (Pintrich, Smith, Garcia \& McKeachie, 1993), and internal consistency, to assess 5 of the 6 motivation self-regulation strategies considered in the original instrument. The final Spanish version of the instrument is a useful tool for conducting further research on this topic. Further studies should select larger samples of college university students attending both public and private universities while also conducting analyses of the instrument's predictive validity.
\end{abstract}

Keywords: self-regulation, motivation, college students, validity, internal consistency

En el aula de clase y fuera de esta los estudiantes en todos los niveles educativos se exponen a tareas académicas en las que, debido a diferentes factores, pueden sentirse aburridos o frustrados, por lo que persistir y comprometerse en estas tareas se hace difícil para ellos, lo que puede desencadenar fallos en su desempeño y aprendizaje (Wolters, 1998, 1999). No obstante, los estudiantes autorregulados persisten y, en el mejor de los casos, superan su falta de motivación, a través de pensamientos y acciones que les permiten controlar activamente su compromiso y disposición para trabajar en sus actividades académicas. El control activo sobre la motivación en las tareas se denomina regulación de la motivación e implica que el estudiante: (a) sea consciente de las creencias que posee sobre los contenidos y tareas que enfrenta y de las razones por las cuales se involucra en ellas; (b) identifique las variaciones en su estado motivacional al enfrentar las actividades académicas y (c) sea capaz de implementar acciones para mantener o aumentar su nivel de motivación, con el fin de persistir en actividades académicas y lograr exitosamente sus objetivos (Wolters, 2003, 2011). De acuerdo con Wolters (2003, 2011), la consciencia e identificación de variaciones sobre los estados motivacionales incrementa las posibilidades de implementación de acciones sobre la motivación; de

Tatiana Rojas-Ospina y Marcela Valencia-Serrano, Departamento de Ciencias Sociales, Facultad de Humanidades y Ciencias Sociales, Pontificia Universidad Javeriana, Cali, Colombia.

La correspondencia relativa a este artículo debe ser dirigida a Tatiana Rojas-Ospina, Departamento de Ciencias Sociales, Pontificia Universidad Javeriana, Calle 18 N 118-250, Edificio El Samán Oficina 3-66, Cali, Valle, Colombia, 760031. E-mail: ctrojas@javerianacali.edu.co 
allí su importancia. Estas acciones encaminadas a mantener o aumentar el nivel de motivación en tareas académicas son denominadas estrategias de autorregulación de la motivación (Sansone, Fraughton, Zachary, Butner \& Heiner, 2011; Wolters, 1998).

En sus inicios, la autorregulación de la motivación fue estudiada sin hacer distinción de otros conceptos, como creencias motivacionales y estrategias de aprendizaje, y fue medida con instrumentos construidos (entrevistas, autorreportes) para indagar estrategias de autorregulación en general. Por ejemplo, los estudios de Purdie y Hattie (1996), Sanson, Weir, Harpster y Morgan (1992) y Zimmermann y Martinez-Pons (1986, 1990) se enfocaron en conocer las estrategias de autorregulación del aprendizaje que usaban estudiantes de secundaria al enfrentar tareas académicas. Se solicitó a los estudiantes dar cuenta de acciones que realizaban para mantener su motivación al ejecutar actividades académicas poco interesantes para ellos, encontrando que los estudiantes reportaron el uso de estrategias, como auto-consecuencias, aumento del interés situacional y estructuración ambiental, para persistir en las tareas.

Más adelante, Wolters (1998) estudió la autorregulación de la motivación como un concepto diferente de las estrategias de aprendizaje y de los procesos motivacionales, centrándose en describir qué estrategias de autorregulación de la motivación utilizaban los estudiantes universitarios. Para esto, desarrolló y aplicó una entrevista semi-estructurada, preguntando a los estudiantes sobre lo que hacían para mantenerse motivados y persistir cuando una tarea académica les parecía aburrida, irrelevante o difícil de realizar. A partir de este estudio fue posible identificar alrededor de 12 estrategias de autorregulación de la motivación.

A partir de estos hallazgos, Wolters (1999) y Wolters y Rosenthal (2000) realizaron investigaciones en las que diseñaron y validaron un cuestionario de autorreporte, que aplicaron a estudiantes de secundaria, para medir la autorregulación de la motivación con cuatro estrategias: auto-consecuencias, estructuración ambiental, incremento del interés y diálogo orientado al dominio y al desempeño. Estas estrategias mostraron relaciones con el desempeño académico, con las estrategias de aprendizaje y con variables motivacionales, como la autoeficacia.

Los estudios mencionados (Wolters, 1998, 1999; Wolters \& Rosenthal, 2000), al centrarse específicamente en estudiar la autorregulación de la motivación, proporcionaron evidencia sólida para establecer que la autorregulación de la motivación es un componente clave del aprendizaje autorregulado, diferente de los procesos motivacionales y de las estrategias cognitivas y metacognitivas. No obstante, la investigación en psicología educativa se ha centrado en estudiar y construir un acervo de conocimiento importante sobre otros componentes del aprendizaje autorregulado, como los procesos motivacionales, las estrategias de aprendizaje y la metacognición, prestando poca atención al estudio de la autorregulación de la motivación (Wolters, 1999, 2003).

Por tanto, diferentes autores plantean que este tema debe ser estudiado como un concepto diferenciado de los demás componentes del aprendizaje autorregulado y que debe ser medido con instrumentos específicos y válidos para que logre consolidarse evidencia sobre su relación con el aprendizaje y el desempeño de los estudiantes en tareas académicas (Paulino, Sá \& Lopes da Silva, 2015a; Schwinger, von der Laden \& Spinath, 2007; Wolters, 2003; Wolters \& Benzon, 2013; Wolters, Benzon \& Arroyo-Giner, 2011).

La diferenciación entre estos componentes del aprendizaje autorregulado está en que las estrategias cognitivas están orientadas a la organización, almacenamiento, elaboración o personalización de los contenidos implicados en las tareas, las estrategias metacognitivas se orientan a la planeación y supervisión del uso de las estrategias cognitivas, los procesos motivacionales dan información sobre los estados motivacionales (interés, expectativas, gusto) de los estudiantes y la autorregulación de la motivación es el componente del aprendizaje autorregulado que explicaría cómo los estudiantes mantienen y elevan sus estados motivacionales durante la tarea y cómo persisten y se esfuerzan en el uso de estrategias cognitivas y metacognitivas para lograr las demandas de esta (Navea-Martín \& Suárez-Riveiro, 2017; Paulino \& Lopes da Silva, 2011; Sansone et al., 2011; Wolters, 2003, 2011).

En este sentido, la literatura muestra un interés creciente por estudiar la autorregulación de la motivación como un componte diferenciado del aprendizaje autorregulado, así como por desarrollar instrumentos para su medición. Por ejemplo, en España Navea-Martín y Suárez-Riveiro (2017), con una muestra de 228 estudiantes universitarios, realizaron un análisis factorial exploratorio (AFE) para el Cuestionario de Estrategias Motivacionales de Aprendizaje, compuesto por cinco factores y originalmente diseñado para estudiantes de secundaria por Suárez y Fernández (2011). El AFE en la muestra de universitarios agrupó los ítems en los mismos cinco factores (estrategia de metas de aprendizaje, de autorefuerzo, de expectativas positivas, de valoración de costes y de pesimismo defensivo), observándose que los 
estudiantes reportaron usar con mayor frecuencia la estrategia de metas de aprendizaje, de auto-refuerzo y de expectativas positivas, mientras que el pesimismo defensivo fue reportado como poco utilizado por los estudiantes.

Por otra parte, la literatura muestra un interés en la definición conceptual y operacional que Wolters (1998, 1999) y Wolters y Rosenthal (2000) realizan de la autorregulación de la motivación, encontrándose dos tendencias de estudios basadas en esas definiciones. La primera tendencia presenta investigaciones que, basadas en las estrategias de autorregulación que proponen Wolters y Rosenthal (2000), han desarrollado sus propios instrumentos para medir las estrategias en estudiantes universitarios (Järvela \& Järvenoja, 2011). La segunda tendencia incluye estudios de validación y adaptación del instrumento desarrollado por Wolters y Rosenthal (2000), con el fin de estudiar las estrategias de autorregulación de la motivación en estudiantes de secundaria (Fritea \& Fritea, 2013; Paulino, Sá \& Lopes da Silva, 2015b; Schwinger, Steinmayr \& Spinath, 2009; Schwinger \& Stiensmeier-Pelster, 2012) y universitarios (Schwinger, Steinmayr \& Spinath, 2012; Wolters \& Benzon, 2013).

Como ejemplo de la primera tendencia, está el estudio de Järvela y Järvenoja (2011), realizado con estudiantes universitarios fineses, cuyo propósito fue estudiar la regulación de la motivación grupal de estudiantes universitarios al enfrentar diferentes retos (comunicación y prioridades personales, entre otros) que están presentes en tareas que involucran trabajo en grupo. Para lograr esto los autores desarrollaron el instrumento Adaptativo para la Regulación de las Emociones y Motivación (AIRE), el cual en una de sus dimensiones evalúa las estrategias que los estudiantes llevan a cabo, tanto individual como grupalmente, para regular sus emociones y motivación durante las tareas. Esta dimensión del AIRE retoma algunas estrategias de regulación de la motivación planteadas por Wolters y Rosenthal (2000), tales como aumento del interés situacional y diálogo orientado a las metas, y, además, añade otras estrategias, como el manejo colectivo de la autoeficacia, la estructuración de la tarea y el reforzamiento social. El estudio concluye que es posible regular la motivación socialmente y que las acciones que se realizan para este fin dependen del tipo de retos que los grupos se planteen.

En cuanto a la segunda tendencia, se encuentra en Alemania la investigación de Schwinger et al. (2007), quienes adaptaron y validaron el Autorreporte de Estrategias de Autorregulación de la Motivación, desarrollado por Wolters y Rosenthal (2000) en estudiantes de bachillerato. En el estudio se realizó un análisis factorial confirmatorio (AFC) que permitió identificar los siguientes factores para agrupar las estrategias de autorregulación de la motivación reportadas por los estudiantes alemanes: aumento de interés situacional, incremento del significado personal, diálogo orientado a metas de desempeño, diálogo orientado a metas de domino, diálogo orientado a metas de desempeño por evitación, auto-consecuencia/reforzamiento, establecimiento de metas proximales y control ambiental.

Además, el instrumento Autorreporte de Estrategias de Autorregulación de la Motivación fue empleado con estudiantes de educación secundaria y superior en Alemania para describir las estrategias utilizadas por los estudiantes para regular su motivación y estimar su relación con variables como la inteligencia, así como su poder predictivo sobre el esfuerzo, la persistencia y el rendimiento académico. Los resultados muestran que las estrategias de autorregulación de la motivación, específicamente las estrategias de regulación de metas de dominio y el establecimiento de metas proximales son predictores importantes del esfuerzo y la persistencia de los estudiantes en tareas académicas (Schwinger et al., 2009; Schwinger et al., 2012; Schwinger \& Stiensmeier-Pelster, 2012).

Asimismo, en Rumania Fritea y Fritea (2013) realizaron una adaptación del Autorreporte de Estrategias de Autorregulación de la Motivación de Wolters y Rosenthal (2000), aplicando a estudiantes de bachillerato cinco de sus escalas con el fin de observar su relación con el aburrimiento y el rendimiento académico: (a) regulación de metas desempeño, (b) regulación del interés situacional, (c) auto-consecuencias, (d) estructuración ambiental y (e) regulación del valor. Los resultados mostraron que las escalas del instrumento se correlacionaban entre sí, que las estrategias de autorregulación de la motivación se correlacionaban inversamente con el aburrimiento y que parecen tener un impacto indirecto sobre el rendimiento de los estudiantes.

Recientemente, en Estados Unidos, Wolters y Benzon (2013) evaluaron el Cuestionario de Estrategias de Autorregulación de la Motivación, tomando en cuenta los estudios de Schwinger et al. (2007) y Schwinger et al. (2009), en una muestra de 215 estudiantes universitarios norteamericanos. Se realizó un AFE, que permitió agrupar las estrategias de autorregulación de la motivación en seis escalas: (a) regulación de valor, (b) regulación de metas de desempeño, (c) auto-consecuencias/auto-refuerzos, (d) estructuración ambiental, (e) regulación del interés situacional y (f) regulación de metas de aprendizaje o dominio (ver Tabla 1). 
Igualmente, se encontró que estas estrategias presentaron correlaciones con estrategias cognitivas y metacognitivas que los estudiantes despliegan al enfrentar tareas académicas, con la autoeficacia y con el valor que estos les dan a las tareas académicas.

Tabla 1

Estrategias de Regulación de la Motivación Desarrolladas en la Literatura

\begin{tabular}{ll}
\hline \multicolumn{1}{c}{$\begin{array}{c}\text { Estrategias de regulación de la } \\
\text { motivación }\end{array}$} & \multicolumn{1}{c}{ Definición } \\
\hline Regulación de valor & $\begin{array}{l}\text { Esfuerzos de los estudiantes por hacer más probable trabajar en sus actividades académicas, } \\
\text { al hacer parecer el material de clase más útil, interesante o importante de conocer. }\end{array}$ \\
Regulación de metas de desempeño & $\begin{array}{l}\text { Esfuerzos por resaltar la importancia de hacerlo bien o de obtener buenas calificaciones en } \\
\text { las actividades académicas que se deben terminar. }\end{array}$ \\
Auto-consecuencias/auto-refuerzos & $\begin{array}{l}\text { Uso de recompensas auto suministradas por el estudiante, como forma de empujarse a sí } \\
\text { mismo a terminar sus trabajos académicos. }\end{array}$ \\
Estructuración ambiental & $\begin{array}{l}\text { Esfuerzos de los estudiantes por controlar aspectos del contexto físico o personal. } \\
\text { Regulación del interés situacional }\end{array}$ \\
Regulación de metas de dominio o & $\begin{array}{l}\text { Intentos del estudiante por hacer que terminar su trabajo académico sea algo más agradable, } \\
\text { divertido o, incluso, como si fuese un juego. } \\
\text { Esfuerzos de los estudiantes por resaltar su deseo de mejorar su comprensión o de aprender } \\
\text { te aprendizaje }\end{array}$ \\
\hline
\end{tabular}

Por último, Paulino et al. (2015b) se propusieron estudiar la relación entre las estrategias de autorregulación de la motivación y las creencias motivacionales. Para esto evaluaron en una muestra de estudiantes de secundaria un inventario que tenía dos dimensiones, creencias motivacionales y estrategias de motivación; la última dimensión se basó en las escalas del instrumento adaptado por Wolters y Benzon (2013). El AFE para la dimensión de estrategias de motivación arrojó cinco factores: (a) interés situacional, (b) regulación del valor, (c) regulación de metas de desempeño, (d) estructuración del contexto y (e) autoconsecuencias, los cuales obtuvieron un alfa de Cronbach entre 0,8 y 0,9. El factor de regulación de metas de aprendizaje no se tuvo en cuenta en el análisis. Los resultados mostraron que las estrategias más usadas por los estudiantes fueron la regulación de metas de desempeño y la estructuración ambiental.

Lo mencionado muestra que los estudios sobre regulación de la motivación se encuentran principalmente en Estados Unidos y Europa y se han centrado en diseñar y validar instrumentos de medida de esta variable y en dar cuenta de sus relaciones con el rendimiento académico y otras variables motivacionales, tales como el esfuerzo, las metas académicas y la autoeficacia, tanto en estudiantes de bachillerato (Paulino et al., 2015b; Schwinger et al., 2007; Schwinger et al. 2009; Schwinger \& Stiensmeier-Pelster, 2012; Wolters, 1999; Wolters \& Rosenthal, 2000) como en estudiantes universitarios (Järvela \& Järvenoja, 2011; Navea-Martín \& SuárezRiveiro, 2017; Schwinger et al., 2012; Wolters, 1998; Wolters \& Benzon, 2013).

Pese a los avances en la investigación sobre la autorregulación de la motivación, que han permitido distinguirla de otros conceptos, como las estrategias de aprendizaje, y que la sitúan como un concepto relevante para comprender el abordaje que los estudiantes hacen de tareas académicas, en Latinoamérica y específicamente en Colombia no existen instrumentos para estudiar la autorregulación de la motivación. Además, los estudios para explicar los procesos cognitivos y motivacionales involucrados en el aprendizaje y desempeño académico de los estudiantes han estado más centrados en analizar las creencias motivacionales, como la orientación a metas y la autoeficacia, y las estrategias de aprendizaje que despliegan los estudiantes en sus actividades académicas, que en las estrategias que usan los estudiantes para regular su motivación y en la influencia de estas en otras dimensiones del aprendizaje autorregulado y en variables como el rendimiento académico (Contreras et al., 2005; Montes, Ayala \& Atencio, 2005; Rinaudo, Chiecher \& Donolo, 2003; Sabogal Tinoco, Barraza Heras, Hernández Castellar \& Zapata, 2011; Valencia Serrano, Duarte Soto \& Caicedo Tamayo, 2013).

Indagar en las estrategias que usan los estudiantes para persistir en la realización de actividades académicas y la relación de estas con otras variables cognitivas y emocionales permitiría construir conocimiento sólido que sustente intervenciones dirigidas a docentes, con el fin de favorecer en ellos el desarrollo de estrategias instruccionales que promuevan el desarrollo, fortalecimiento y/o mantenimiento de 
las estrategias de autorregulación de motivación en los estudiantes, de tal manera que tengan recursos que les posibiliten superar las diversas dificultades que enfrentan para persistir y mantener el interés en las actividades que abordan. Asimismo, la evidencia que logre consolidarse sobre el tema también contribuiría a la construcción de programas psicoeducativos enfocados en enseñar a los estudiantes formas de monitorear sus estados motivacionales al abordar tareas académicas, así como estrategias para regularlos y persistir en estas. Lo anterior puede aportar también en el abordaje de la problemática de deserción estudiantil en la educación superior, que en 2015 alcanzó el 46\% en Colombia (Ministerio de Educación Nacional, 2015) y que en los primeros semestres de I a V año alcanzó entre el 37\% y 40\% en las diferentes áreas del conocimiento (Melo-Becerra, Ramos-Forero \& Hernández-Santamaría, 2017).

Tomando en cuenta lo mencionado, este estudio se planteó como objetivo adaptar el instrumento diseñado por Wolters y Benzon (2013) y evaluar sus propiedades psicométricas (validez de divergente y discriminante y consistencia interna) en una muestra de estudiantes universitarios colombianos, a través de un AFE y un AFC.

\section{Método}

\section{Participantes}

Se seleccionaron intencionalmente 315 estudiantes universitarios, pertenecientes a dos universidades privadas de la ciudad de Cali, Colombia (51\% mujeres y 49\% hombres), con una edad promedio de 19 años $(D E=3,06, \operatorname{Min}=16$, Máx $=42)$. Los estudiantes se encontraban cursando entre $1^{\circ}$ y $5^{\circ}$ semestre de carreras de las siguientes áreas: Ingeniería (36\%), Ciencias económicas y administrativas (42\%) y Humanidades (22\%). Los criterios de inclusión fueron: (a) estar cursando entre $1^{\circ}$ y $5^{\circ}$ semestre, considerando que es en estos semestres en los que se presentan mayores índices de deserción en Colombia, y (b) que los participantes reportaran haberse sentido desmotivados en alguna asignatura que estuviesen cursando durante el semestre en el que se administraron los instrumentos.

\section{Instrumentos}

Cuestionario de Estrategias de Autorregulación de la Motivación. Fue diseñado por Wolters y Benzon (2013) para evaluar las acciones que los estudiantes universitarios realizan para mantener o elevar su motivación en situaciones académicas. El cuestionario consta de 30 ítems que se responden en una escala tipo Likert de 7 puntos, desde 1 (Nunca) a 7 (Muy frecuentemente). El cuestionario consta de seis factores o escalas: (a) Auto-Consecuencias (5 ítems; e.g., Me digo a mí mismo que puedo hacer algo que me gusta después, si ahora mismo hago el trabajo que tengo que tener listo), (b) Estructuración Ambiental (4 ítems; e.g., Trato de deshacerme de cualquier distracción que esté a mi alrededor), (c) Regulación del Valor de la Tarea (6 ítems; e.g., Trato de hacerme ver a mí mismo que conocer el material es personalmente relevante), (d) Regulación de Metas de Desempeño (5 ítems; e.g., Me recuerdo a mi mismo lo importante que es obtener buenas calificaciones), (e) Regulación del Interés Situacional (5 ítems; e.g., Hago el estudio más agradable convirtiéndolo en un juego) y (f) Regulación de Metas de Aprendizaje (5 ítems; e.g., Me digo a mí mismo que debo estudiar solo para aprender tanto como pueda). En la validación realizada por Wolters y Benzon (2013) se llevó a cabo un AFE, el cual arrojó cargas factoriales aceptables de los ítems en cada factor, excepto un elemento que fue eliminado. Además, las escalas del instrumento presentaron una alta consistencia interna, con alfa de Cronbach entre 0,77 y 0,91 . El instrumento fue traducido al español y adaptado para estudiantes universitarios colombianos.

Cuestionario de Estrategias Motivacionales y de Aprendizaje (MSLQ por las siglas en inglés del Motivated Strategies for Learning Questionnaire). Este cuestionario fue diseñado por Pintrich, Smith, Garcia y McKeachie (1993) para evaluar la motivación y el uso de estrategias de aprendizaje de estudiantes universitarios. El cuestionario se compone de 81 ítems en dos secciones: motivación (31 ítems) y estrategias de aprendizaje (50 ítems). Los ítems del cuestionario se responden en una escala tipo Likert de 7 puntos, desde 1 (Nada verdadero para mí) a 7 (Muy verdadero para mî). Para este estudio se usaron cinco escalas de la sección de motivación pertenecientes a los componentes de valor y expectativas. Del componente de valor se aplicaron las escalas: (a) Orientación al Desempeño (4 ítems; e.g., Obtener una buena calificación en esta clase es lo más satisfactorio para mí ahora), (b) Orientación al Aprendizaje (4 ítems; e.g., En una clase como esta, prefiero el material de la asignatura que realmente me desafía, porque así puedo aprender nuevas cosas) y (c) Valoración de la Tarea (6 ítems; e.g., Pienso que seré capaz de usar en otros cursos lo que aprenda 
en este curso) y del componente de expectativas se aplicaron las escalas: (a) Creencias de Autoeficacia (8 ítems; e.g., Creo que recibiré una excelente calificación en esta clase) y (b) Creencias de Control del Aprendizaje (4 ítems; e.g., Es mi culpa si no aprendo el material en este curso). En el estudio de validación de Pintrich et al. (1993) se realizó un AFC y un análisis de confiabilidad con alfa de Cronbach, presentando una consistencia interna entre 0,64 y 0,93 .

En este estudio, los ítems de las escalas Valoración de la Tarea y Orientación al Aprendizaje fueron tomados del estudio de validación del MSLQ que realizaron Sabogal-Tinoco et al. (2011) en una muestra de estudiantes universitarios de Santa Marta, Colombia. En dicho estudio, el AFE mostró acuerdo con la estructura original presentada por Pintrich et al. (1993) y los ítems de las escalas presentaron una consistencia interna, evaluada con alfa de Cronbach, entre 0,83 y 0,85. El resto de las escalas (Orientación al Desempeño, Creencias de Autoeficacia, Creencias de Control del Aprendizaje) se sometieron a un proceso de traducción-retraducción del original.

\section{Procedimiento}

El Cuestionario de Estrategias de Autorregulación de la Motivación pasó por un proceso de adaptación al español que constó de dos momentos: el primero consistió en una traducción inversa (Hambleton, 1994; Hambleton \& Kanjee, 1995). Inicialmente la versión original del cuestionario se tradujo al castellano por un psicólogo nativo colombiano con maestría en educación. Dicha traducción fue revisada por otro psicólogo nativo colombiano, magister en psicología y con conocimiento de ambos idiomas. Una vez que se tradujo el instrumento, la versión en español fue traducida de nuevo al inglés por dos traductores nativos en esta lengua y conocedores de ambas culturas: uno de ellos profesor de idiomas y el otro psicólogo. Luego, se comparó la versión original y la traducida del instrumento para realizar ajustes finales a los ítems.

Dado que Sabogal Tinoco et al. (2015) no validaron los ítems de las escalas Orientación al Desempeño, Creencias de Autoeficacia y Creencias de Control del Aprendizaje del MSLQ, se realizó el mismo proceso de traducción-retraducción mencionado para el Cuestionario de Estrategias de Autorregulación de la Motivación.

Los ítems de ambos instrumentos fueron sometidos a un estudio piloto con 42 estudiantes universitarios, con el fin de afinar la redacción y comprensión de los ítems. No se eliminaron ítems ni se realizaron cambios sustanciales en los instrumentos después del estudio piloto.

La versión final del instrumento que se aplicó a los participantes con ambos cuestionarios, se presentó en formato electrónico, acompañada del consentimiento informado para participar en la investigación, en el cual se aclaró a los estudiantes que su participación era voluntaria y que los datos eran anónimos y confidenciales. Los estudiantes declaraban por escrito si accedían a participar. El instrumento se aplicó durante el primer semestre del año, aproximadamente tres semanas después de la realización de los primeros exámenes parciales. Su aplicación se realizó en los salones de clase y tomó aproximadamente 45 minutos.

\section{Análisis de Datos}

Para analizar la distribución de las puntuaciones del cuestionario de estrategias de autorregulación de la motivación se calcularon los estadísticos de asimetría y curtosis para el caso de normalidad univariada. La normalidad multivariada se evaluó mediante el coeficiente de curtosis normalizada de Mardia.

Se realizó un AFE, evaluando las características de las matrices, por medio del test de esfericidad de Bartlett y, la medida de Kaiser-Meyer-Olkin (KMO) de adecuación de la muestra. Cuanto más cercano a 1 sea el valor obtenido de KMO, se considera positivo e indica que se puede continuar con el AFE. También se calcularon las medidas individuales de adecuación de la muestra (MSA), en la que valores superiores a 0,80 se consideran meritorios de realizar un AFE, de acuerdo con Kaiser (1974), citado en Pett, Lackey y Sullivan (2003). Los ítems se analizaron por medio de rotación oblicua. Este análisis se enfoca en el factor latente cuando se identifica la varianza común entre los ítems (Henson \& Roberts, 2006). Se usó la rotación oblicua, considerando que se asume que los factores son dimensiones correlacionadas del constructo autorregulación de la motivación (Pett et al., 2003). En este análisis se examinó la tabla de comunalidades, aceptando valores superiores a 0,40 , lo cual indica que mucha de la varianza común en los ítems puede ser explicada por los factores extraídos. Posteriormente, con el fin de identificar los factores a extraer, se consideró el criterio Kaiser del valor propio (eigenvalue) mayor a 1, la observación del gráfico de sedimentación, el Minum Average Partial Test (MAP) propuesto por Velicer (1976), la significancia teórica de los factores y los resultados del 
análisis exploratorio realizado previamente por el autor del instrumento en su versión en inglés (Wolters \& Benzon, 2013).

Los siguientes criterios fueron usados con el fin de determinar los factores y los ítems que estarían o no en la solución final: (a) debía haber al menos dos ítems con cargas altas en la matriz de patrones para identificar un factor (Netemeyer, Bearden \& Sharma, 2003), (b) se retuvieron ítems con coeficientes de patrón superiores a 0,4 y (c) se evaluó si existía multidimensionalidad, en cuyo caso se eliminarían aquellos ítems con cargas altas en al menos dos factores (Pett et al., 2003).

Luego, se realizó un AFC, retomando para ello el modelo producto del AFE realizado previamente, y se estimó en qué medida el modelo observado en el AFE se ajustaba a los datos de la muestra. Este análisis se realizó con la corrección robusta de Satorra-Bentler (S-B; Bentler, 2006), con el fin de controlar la posible incidencia de la no normalidad multivariada de los datos. Se utilizaron los siguientes criterios, propuestos por Netemeyer et al. (2003), para evaluar el modelo: (a) convergencia del modelo y un rango aceptable de los parámetros estimados, los cuales incluyen: varianzas positivas de los ítems y cargas factoriales, así como correlaciones inferiores a 1; (b) los resultados del estadístico $\chi^{2}$ que prueba el modelo nulo frente al modelo propuesto. De no resultar significativo, se interpreta como un adecuado ajuste del modelo a los datos. Sin embargo, según McIntosh (2007), $\chi^{2}$ es susceptible al tamaño de la muestra y además requiere de normalidad multivariada; de lo contrario, puede generar un rechazo del modelo, aunque este se encuentre adecuadamente especificado. Por este motivo, se incorporó también la razón entre $\chi^{2}$ y los grados de libertad $\left(\chi^{2} / g l\right)$, la cual se considera un buen indicador si el resultado oscila entre 1 y 3 (Carmines \& McIver, 1981). También se incluyó la raíz del error cuadrático medio de aproximación (RMSEA), considerando un modelo aceptable si se obtienen valores inferiores 0,06 (Schreiber, Nora, Stage, Barlow \& King, 2006). Además, se consideraron medidas de ajuste comparativo, como el índice de Tucker Lewis (TLI) y el índice comparativo de Bentler-Bonett (CFI), en los que valores cercanos a 0,95 son considerados aceptables (Hu \& Bentler, 1999; Netemeyer et al., 2003), y el índice comparativo entre modelos, el criterio de información de Akaike (AIC), para el cual valores cercanos a 0 indican un mejor ajuste.

Se usó el programa estadístico IBM SPSS v.23 y el programa estadístico Stata/IC 15.0 para la realización del AFE y el AFC, respectivamente.

Posteriormente, se realizó un análisis de consistencia interna de cada escala, por medio del cálculo de alfa de Cronbach. Se examinaron las matrices de correlaciones entre ítems de cada escala, buscando valores mayores a 0,30, así como alfa de Cronbach si el ítem era eliminado.

Finalmente, se analizó la validez convergente y discriminante, correlacionando los resultados del cuestionario de estrategias de autorregulación de la motivación con los obtenidos en el MSLQ de Pintrich et al. (1993).

\section{Resultados}

Las puntuaciones del cuestionario de estrategias de autorregulación de la motivación presentaron estadísticos de asimetría y curtosis que variaron de -0,026 (ítem 6) a -1,318 (ítem 22) y de -0,088 (ítem 23) a -1,432 (ítem 6), respectivamente, indicando la presencia de normalidad univariada. El valor del coeficiente de curtosis normalizada multivariado de Mardia fue de 150,60 para todos los ítems, indicando la presencia de no-normalidad multivariada severa en la distribución de las puntuaciones.

$\mathrm{Al}$ realizar el AFE, en primera instancia fueron evaluadas las características de las matrices. La matriz de correlación entre ítems mostró correlaciones superiores a 0,30 en todos los ítems (solo hubo una correlación mayor a 0,80 entre los ítems 27 y 28). El test de esfericidad de Bartlett, $\chi^{2}(435, N=315)=5265,074$, $p<0,001$, fue significativo. La medida de KMO de adecuación de la muestra fue de 0,913 y las medidas de MSA fueron superiores a 0,80 en todos los ítems. Estos test permitieron concluir que la matriz de datos era factorizable.

Se examinó la tabla de comunalidades, reportando altas comunalidades en 28 de 30 ítems $(>0,40)$, excepto por dos ítems que presentaron bajas comunalidades $(0,20>0,40)$, los que fueron eliminados para los análisis posteriores.

Siguiendo los criterios establecidos, se identificó que la solución de cinco factores era la más aceptable. Juntos, los cinco factores dan cuenta del $54,3 \%$ de la varianza. Esta solución presentó ítems con cargas superiores a 0,50 en al menos un factor que tenía sentido teóricamente, por tanto, fueron retenidos. Sin embargo, tres ítems presentaron cargas inferiores a 0,40; por consiguiente, fueron eliminados para los análisis posteriores. Los resultados de estos análisis, incluyendo los valores propios (eigenvalues) y las cargas de los factores de la matriz de patrones, son presentados en la Tabla 2. Los factores extraídos fueron cinco: 
Regulación del Valor de la Tarea (8 ítems), Regulación de Metas de Desempeño (5 ítems), Auto-Consecuencias (5 ítems), Estructuración Ambiental (4 ítems) y Regulación del Interés Situacional (5 ítems).

Posteriormente, se realizó el AFC con base en la estructura factorial arrojada por el análisis previo. El modelo de cinco factores inicialmente probado (ver Figura 1) no presentó un buen ajuste del modelo a los datos, $\chi^{2}(314, N=315)=790,365, p<0,001 \chi^{2} / g l=2,52$, RMSEA $=0,069$, TLI $=0,861$, CFI $=0,845$ y AIC $=29418,249$. Por consiguiente, se examinaron las covarianzas estandarizadas residuales de los ítems, las cuales, según Fernández Aráuz (2015), pueden ser entendidas como "el número de desviaciones estándar por el cual los residuos ajustados difieren de los residuos de valor cero que estarían asociados con un modelo de ajuste perfecto" (p. 58) y, por tanto, pueden generar que se subestime o sobreestime la relación entre los indicadores. Al revisar estos valores, se encontraron valores altos $(>2,56)$ entre los residuos de los ítems 8 y 18 del factor Regulación de Metas de Desempeño, de los residuos de los ítems 6 y 10 del factor AutoConsecuencias y de los residuos de los ítems 5 y 13 del factor Regulación del Interés Situacional, los cuales fueron correlacionados (ver Figura 2). Adicionalmente, se identificó que los ítems 3, 16, 29 del factor Regulación del Valor de la Tarea, el ítem 9 del factor Estructuración Ambiental y el ítem 4 del factor Regulación del Interés Situacional presentaban valores altos en varios ítems de otros factores, por lo que fueron eliminados. Una vez hechos estos ajustes, el segundo modelo de cinco factores (ver Figura 2) presentó un mejor ajuste a los datos, $\chi^{2}(196, N=315)=348,535, p<0,001, \chi^{2} / g l=1,78$, RMSEA $=0,050$, TLI $=0,931$, $\mathrm{CFI}=0,942$ y $\mathrm{AIC}=23958,140$.

Finalmente, los factores confirmados fueron cinco de los seis inicialmente presentes en la escala, con un total de 22 ítems. Todos los factores presentaron valores adecuados de alfa de Cronbach $(0,70<\alpha<0,80)$. Los datos de consistencia interna de cada una de las subescalas son presentados en la Tabla 3. 
Tabla 2

\section{Estructura Factorial con Rotación Oblicua de la Escala de Estrategias de Autorregulación de la Motivación}

\begin{tabular}{lc}
\hline \multicolumn{1}{c}{ Ítem } & 1 \\
\hline $\begin{array}{l}\text { 16. Me digo a mí mismo que es importante aprender el material porque lo } \\
\text { necesitaré más adelante en mi vida }\end{array}$ & 0,693 \\
$\begin{array}{l}\text { 24. Trato de hacerme ver a mí mismo que conocer el material es personalmente } \\
\text { relevante }\end{array}$ & 0,685 \\
$\begin{array}{l}\text { 25. Trato de lograr que el material parezca más útil, relacionándolo con lo que } \\
\text { quiero hacer en mi vida }\end{array}$ & 0,681 \\
$\begin{array}{l}\text { 3. Hago el esfuerzo de relacionar lo que estamos aprendiendo con mis intereses } \\
\text { personales }\end{array}$ & 0,572 \\
$\begin{array}{l}\text { 21. Pienso en situaciones en las que sería útil para mí conocer el material o las } \\
\text { habilidades }\end{array}$ & 0,535 \\
$\begin{array}{l}\text { 23. Me reto a mí mismo para completar el trabajo y aprender tanto como sea } \\
\text { posible }\end{array}$ & 0,491 \\
$\begin{array}{l}\text { 26. Me persuado a mí mismo de mantenerme estudiando solo para ver qué tanto } \\
\text { puedo aprender }\end{array}$ & 0,472 \\
$\begin{array}{l}\text { 29. Me digo a mí mismo que debo estudiar solo para aprender tanto como pueda } \\
\text { 14. Me digo a mí mismo que debería seguir trabajando solo para aprender tanto } \\
\text { como pueda }\end{array}$ & 0,433 \\
\end{tabular}

30. Trato de conectar el material con algo que me gusta hacer o que encuentro interesante $^{a}$

22. Me recuerdo a mí mismo lo importante que es obtener buenas calificaciones

0,825

0,616

0,577

0,545

0,459

6. Me prometo a mí mismo alguna clase de recompensa si realizo mis lecturas o si estudio

19. Trato de deshacerme de cualquier distracción que esté a mí alrededor

20. Me convenzo a mí mismo de trabajar duro solo en aras del aprendizajea

13. Trato de hacer un juego del aprendizaje del material o de completar la tarea

5. Pienso en una manera de hacer que el trabajo parezca agradable de completar

$\begin{array}{rrrrrr}\text { Eigenvalue } & 10,2 & 2,2 & 1,4 & 1,1 & 0,8 \\ \text { Porcentaje de varianza explicada } & 34,0 & 7,6 & 4,8 & 3,7 & 2,9\end{array}$

Nota. Cargas inferiores a 0,35 no son presentadas. 1 = Factor 1/Regulación del Valor de la Tarea; 2 = Factor 2/Regulación de Metas de Desempeño; $3=$ Factor 3/Auto-Consecuencias; 4 = Factor 4/Estructuración Ambiental; $5=$ Factor 5/Regulación del Interés Situacional.

a ítem no usado para posteriores análisis. 


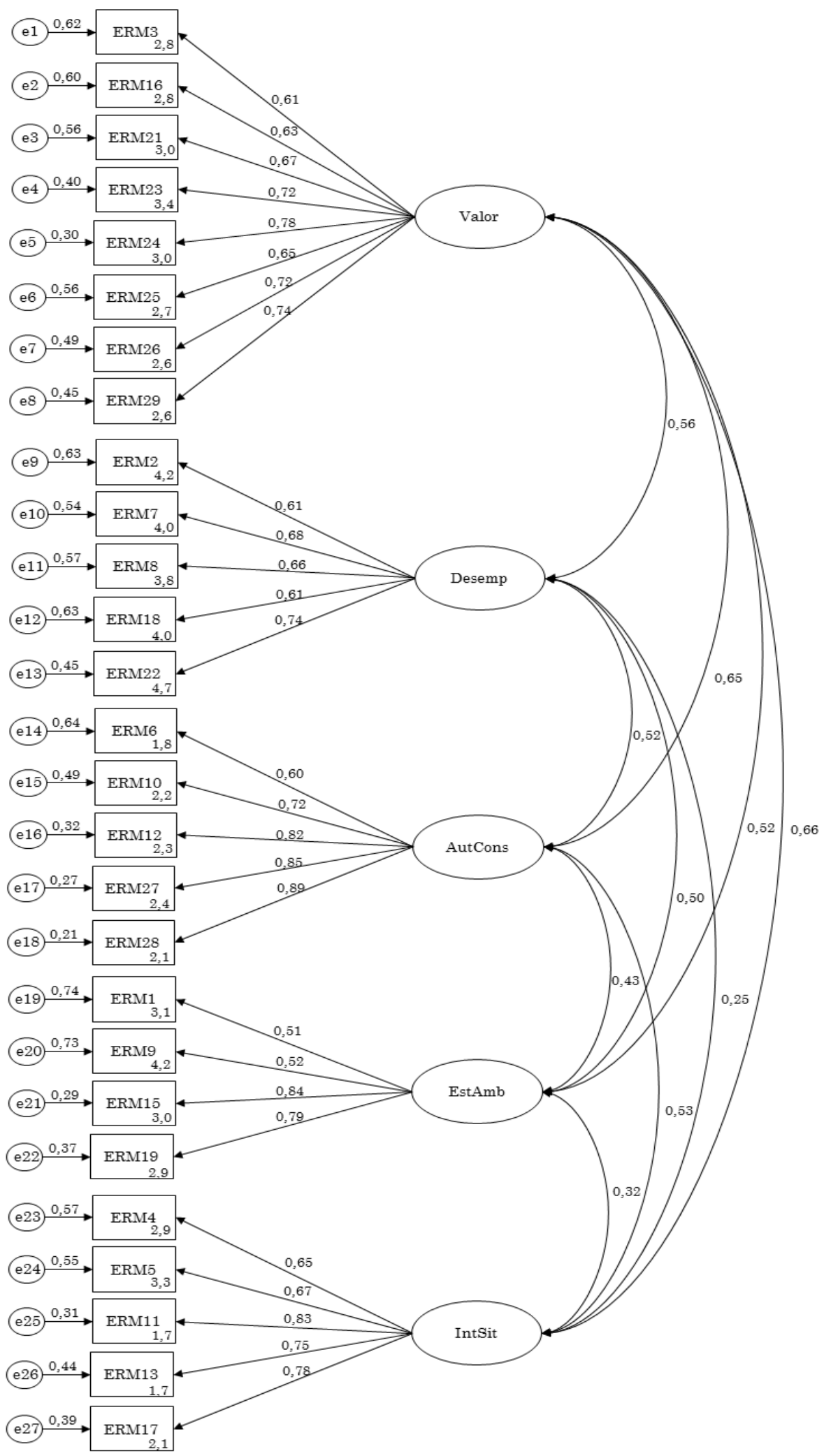

Figura 1. Modelo inicial de cinco factores. Valor = Regulación del Valor de la Tarea; Desemp = Regulación de Metas de Desempeño; AutCons = Auto-Consecuencias; EstAmb = Estructuración Ambiental; IntSit $=$ Regulación del Interés Situacional. Los valores al interior de cada ítem corresponden a los interceptos estandarizados. 

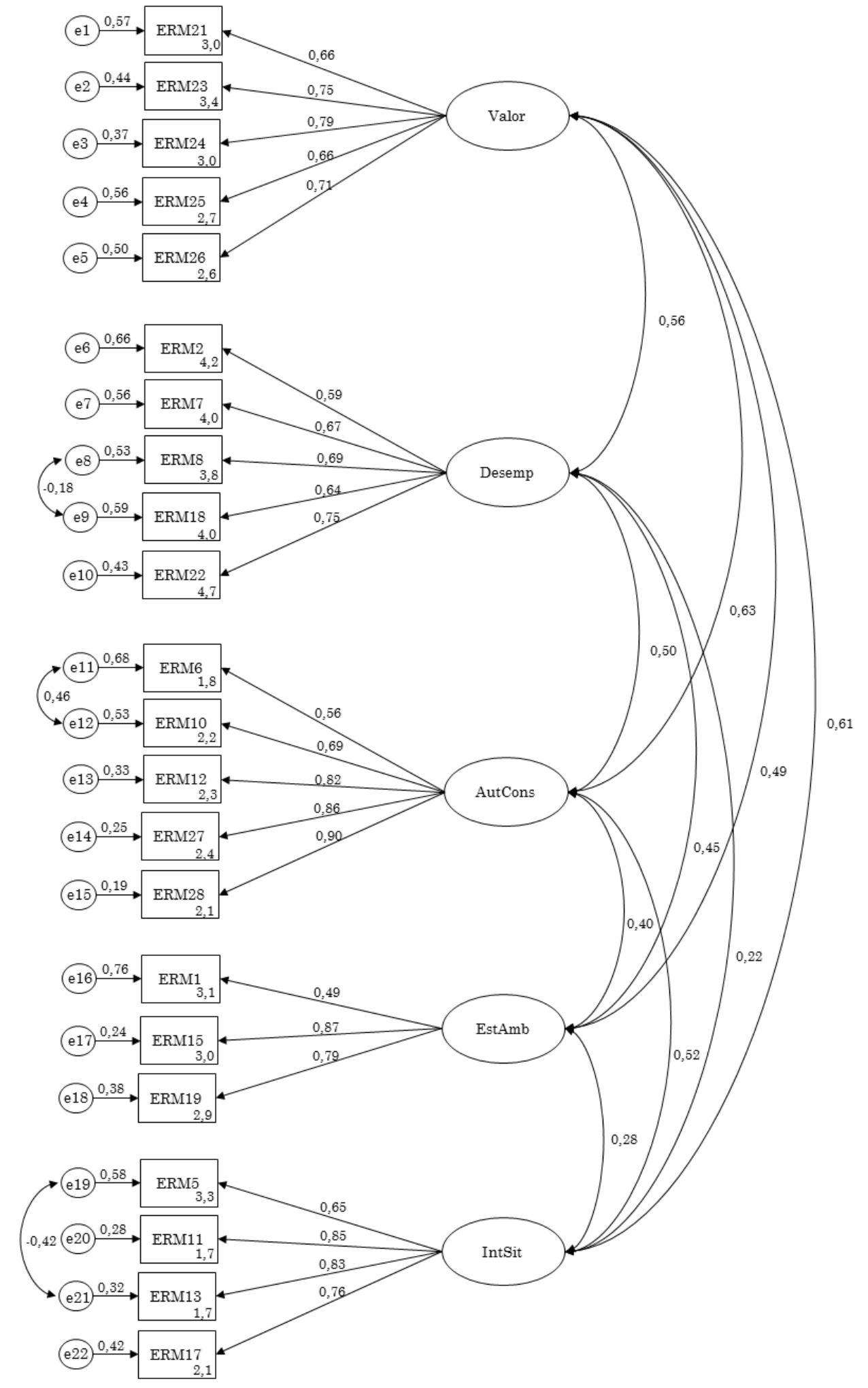

Figura 2. Modelo final de cinco factores. Valor = Regulación del Valor de la Tarea; Desemp = Regulación de Metas de Desempeño; AutCons = AutoConsecuencias; EstAmb = Estructuración Ambiental; IntSit = Regulación del Interés Situacional. Los valores al interior de cada ítem corresponden a los interceptos estandarizados. 
Tabla 3

Índices de Consistencia Interna para las Subescalas del Instrumento $(N=315)$

\begin{tabular}{lccc}
\hline \multicolumn{1}{c}{ Escala } & Cantidad de ítems & $\alpha$ & $95 \%$ IC \\
\hline Regulación del valor de la tarea & 5 & 0,84 & $0,80,0,86$ \\
Regulación de metas de desempeño & 5 & 0,79 & $0,75,0,82$ \\
Auto-consecuencias & 5 & 0,88 & $0,86,0,90$ \\
Estructuración ambiental & 3 & 0,75 & $0,69,0,79$ \\
Regulación del interés situacional & 4 & 0,84 & $0,81,0,87$ \\
\hline
\end{tabular}

En relación con la validez convergente y discriminante, como se observa en la Tabla 4, se evidenciaron correlaciones directas entre las estrategias de Regulación del Valor de la Tarea y la estrategia de Regulación de Metas de Desempeño con todas las creencias motivacionales. Se destacan las correlaciones altas entre la estrategia de Regulación del Valor de la Tarea y las creencias de Valoración de la Tarea y Orientación al Aprendizaje, así como la correlación entre la estrategia de Regulación de Metas de Desempeño y las creencias motivacionales de Orientación al Desempeño. La estrategia de Estructuración Ambiental presentó correlaciones positivas con todas las creencias motivacionales. Finalmente, las estrategias de AutoConsecuencias y Regulación del Interés Situacional presentaron correlaciones positivas con todas las creencias motivacionales, excepto con las creencias de control del aprendizaje.

Tabla 4

Correlaciones entre las Puntuaciones del MSQL y del Cuestionario de Estrategias de Autorregulación de la Motivación (N = 315)

\begin{tabular}{|c|c|c|c|c|c|}
\hline & $\begin{array}{l}\text { Orientación } \\
\text { desempeño }\end{array}$ & $\begin{array}{l}\text { Orientación } \\
\text { aprendizaje }\end{array}$ & $\begin{array}{l}\text { Valoración de } \\
\text { la tarea }\end{array}$ & $\begin{array}{l}\text { Creencias de } \\
\text { control del } \\
\text { aprendizaje }\end{array}$ & $\begin{array}{l}\text { Creencias de } \\
\text { autoeficacia }\end{array}$ \\
\hline $\begin{array}{l}\text { Regulación del valor de la } \\
\text { tarea }\end{array}$ & $0,265^{* *}$ & $0,425^{* *}$ & $0,504^{* *}$ & $0,200^{* *}$ & $0,322^{* *}$ \\
\hline $\begin{array}{l}\text { Regulación de metas de } \\
\text { desempeño }\end{array}$ & $0,484^{* *}$ & $0,266^{* *}$ & $0,216^{* *}$ & $0,147^{* *}$ & $0,218^{* *}$ \\
\hline Auto-consecuencias & $0,236^{* *}$ & $0,279^{* *}$ & $0,276^{* *}$ & 0,029 & $0,227^{* *}$ \\
\hline Estructuración ambiental & $0,130^{*}$ & $0,219 * *$ & $0,196^{* *}$ & $0,127^{*}$ & $0,224^{* *}$ \\
\hline $\begin{array}{l}\text { Regulación del interés } \\
\text { situacional }\end{array}$ & $0,125^{*}$ & $0,312^{* *}$ & $0,352^{* *}$ & 0,050 & $0,281^{* *}$ \\
\hline
\end{tabular}

Nota. ${ }^{* *} p<0,01 ;{ }^{*} p<0,05$.

\section{Discusión y Conclusiones}

El AFE permitió identificar cinco de los seis factores extraídos por Wolters y Benzon (2013). El factor Regulación de Metas de Dominio no fue extraído en el análisis, a diferencia de los resultados de la validación realizada por Wolters y Benzon (2013) con estudiantes universitarios estadounidenses y similar a los resultados presentados por Paulino et al. (2015b) con estudiantes portugueses de secundaria. En el estudio actual no es posible identificar si la no extracción de este factor puede deberse a las características propias de la muestra y de su contexto o a un resultado producto de diferencias culturales de los participantes de los estudios y, por tanto, este se constituye en un asunto para continuar trabajando en futuras investigaciones.

Las correlaciones entre los factores también permiten confirmar la multidimensionalidad del instrumento. Estos datos permiten constatar que los factores son suficientemente independientes entre sí, tal como ha sido identificado en los estudios previos de Wolters y Benzon (2013) y Paulino et al. (2015b). Las correlaciones con mayor magnitud se encuentran entre los factores Regulación del Valor de la Tarea y Auto- 
Consecuencias y Regulación del Valor de la Tarea y Regulación del Interés Situacional. Por otro lado, las correlaciones más bajas se observan entre las estrategias de Regulación de Metas de Desempeño y Regulación del Interés Situacional y Estructuración Ambiental y Regulación del Interés Situacional. Estos resultados coinciden con los presentados por Wolters y Benzon (2013) y pueden ser explicados a partir de los planteamientos de la teoría de la autodeterminación de Ryan y Deci (2000), respecto de las formas de regulación que se encuentran relacionadas con los tipos de motivación, extrínseca o intrínseca. En el caso de la estrategia de Regulación de Metas de Desempeño, el estudiante busca mejorar su motivación al pensar en su necesidad de obtener buenas calificaciones, lo cual es un ejemplo de motivación extrínseca, asociada con una regulación de tipo externo, es decir, que los comportamientos se realizan con el fin de satisfacer una demanda externa o de recibir una recompensa, como en este caso lo serían las calificaciones. A su vez, esto explicaría la baja correlación con la estrategia de Regulación del Interés Situacional que tiene un carácter más intrínseco, pues en esta el estudiante recurre a la búsqueda del disfrute y del placer. Por otro lado, en el caso de la estrategia de Regulación del Valor de la Tarea, aunque identificar la utilidad de la actividad pueda ser considerado como parte de la motivación extrínseca, la forma de regulación utilizada es algo interna, en la cual el estudiante identifica la importancia personal de la tarea y, por tanto, la necesidad de continuar esforzándose en la misma, porque es útil e interesante. Por ello el estudiante buscaría otras formas de motivarse a continuar trabajando a partir de premios que se da a sí mismo y que no provienen de una fuente externa (Auto-Consecuencias) o intentaría hacer la actividad más placentera y divertida (Regulación del Interés Situacional).

En relación con la validez convergente y discriminante, se encontró que la subescala Valoración de la Tarea del MSQL presentó las correlaciones más altas con las diferentes estrategias de autorregulación de la motivación, a excepción de la Regulación de Metas de Desempeño, que presentó la más alta correlación con la subescala de creencias de Orientación al Desempeño del MSQL. Estos resultados son similares a los presentados en los estudios realizados por Wolters (1998) y Wolters y Benzon (2013), respecto del poder predictivo de las creencias motivacionales con las diferentes estrategias de regulación de la motivación. De acuerdo con Wolters $(1998,2003)$, esto se explica porque en la medida en que los estudiantes encuentren útil, importante o personalmente relevante el material de aprendizaje, ellos harán uso de diferentes estrategias para mantener su motivación a lo largo de las actividades académicas. Adicionalmente, en cuanto a las creencias de Valoración de la Tarea, se identificaron valores más altos en las correlaciones con las estrategias de Regulación del Valor de la Tarea y Regulación del Interés Situacional, dando cuenta de nuevo de su carácter más intrínseco. En este mismo sentido, la subescala de creencias de Orientación a Metas de Desempeño, que presenta un carácter más extrínseco, presenta la correlación más alta con la estrategia de Regulación de Metas del Desempeño. Finalmente, los niveles de consistencia interna de los factores identificados fueron adecuados.

En conclusión, el cuestionario sobre estrategias de autorregulación de la motivación en su versión adaptada al castellano con 22 ítems y cinco factores se ajusta a la muestra utilizada. La versión final del cuestionario presenta una adecuada validez convergente y discriminante, lo que es congruente con los resultados de estudios previos (Schwinger et al., 2012; Wolters \& Benzon, 2013), en los que también se encontraron correlaciones entre diferentes variables motivacionales y las estrategias de autorregulación de la motivación. En consecuencia, el instrumento se constituye en una herramienta útil para continuar el estudio de los procesos de control activo que los estudiantes realizan sobre sus propios procesos motivacionales, para de esta forma seguir indagando en la motivación desde la perspectiva del estudiante.

Futuros estudios podrán continuar analizando las propiedades psicométricas del cuestionario con el fin de generar un instrumento que pueda ser adaptado a diferentes poblaciones universitarias en Colombia o en otros países de habla castellana. Con este fin, se hace necesario atender a algunas limitaciones del estudio: (a) generar más ítems para la subescala de Estructuración Ambiental, que en el estudio actual quedó conformada por solo tres ítems, lo que pudo afectar el nivel de confiabilidad alcanzado por esta subescala; (b) seleccionar una muestra más amplia de participantes, que incluya universidades públicas y privadas de todo el país y de otros países latinoamericanos y (c) determinar la validez predictiva del instrumento para establecer si la frecuencia de uso de cada una de las estrategias de autorregulación de motivación podría relacionarse con variables relacionadas con el desempeño académico y con la deserción de los estudiantes en las asignaturas en las que se encuentran desmotivados. Este aspecto es particularmente importante, porque constituiría un foco de intervención en programas académicos y en asignaturas con altos niveles de deserción. 


\section{Referencias}

Bentler, P. M. (2006). EQS 6 Structural equations program manual. Encino, CA: Multivariate Software.

Carmines, E. G. \& McIver, J. P. (1981). Analyzing models with unobserved variables: Analysis of covariance structures. En G. W. Bohrnstedt \& E. F. Borgatta (Eds.), Social measuarment: Current issues (pp. 65-115). Beverly Hills, CA: SAGE. https://doi.org/10.117/0049124183011003003

Colombia, Ministerio de Educación Nacional (2015). Estadisticas deserción y graduación 2015. Bogotá, Colombia: Autor. Extraído de https://www.mineducacion.gov.co/sistemasdeinformacion/1735/articles-357549_recurso_3.pdf

Contreras, F., Espinosa, J. C., Esguerra, G., Haikal, A., Polanía, A. \& Rodríguez, A. (2005). Autoeficacia, ansiedad y rendimiento académico en adolescentes. Diversitas: Perspectivas en Psicología, 1, 183-194. https://doi.org/10.15332/s1794-9998.2005.0002.06

Fernández Aráuz, A. (2015). Aplicación del análisis factorial confirmatorio a un modelo de medición del rendimiento académico en lectura. Revista de Ciencias Económicas, 33(2), 39-66. https://doi.org/10.15517/rce.v33i2.22216

Fritea, I. \& Fritea, R. (2013). Can motivational regulation counteract the effects of boredom on academic achievement? Procedia-Social and Behavioral Sciences, 78, 135-139. https://doi.org/10.1016/j.sbspro.2013.04.266

Hambleton, R. K. (1994). Guidelines for adapting educational and psychological tests: A progress report. European Journal of Psychological Assessment, 10, 229-244. Extraído de https://files.eric.ed.gov/fulltext/ED399291.pdf

Hambleton, R. K. \& Kanjee, A. (1995). Increasing the validity of cross-cultural assessments: Use of improved methods for test adaptations. European Journal of Psychological Assessment, 11, 147-157. https://doi.org/10.1027/1015-5759.11.3.147

Henson, R. K. \& Roberts, J. K. (2006). Use of exploratory factor analysis in published research: Common errors and some comments on improved practice. Educational and Psychological Measurement, 66, 393-416. https://doi.org/10.1177/0013164405282485

Hu, L. \& Bentler, P. M. (1999). Cutoff criteria for fit indexes in covariance structure analysis: Conventional criteria versus new alternatives. Structural Equation Modeling, 6, 1-55. https://doi.org/10.1080/10705519909540118

Järvelä, S., Järvenoja, H. (2011). Socially constructed self-regulated learning and motivation regulation in collaborative learning groups. Teachers College Record, 113(2), 350-374. Extraído de https://eric.ed.gov/?id=EJ927081

McIntosh, C. M. (2007). Rethinking fit assessment in structural equation modelling: A commentary and elaboration on Barrett (2007). Personality and Individual Differences, 42, 859-867. https://doi.org/10.1016/j.paid.2006.09.020

Melo-Becerra, L. A., Ramos-Forero, J. E. \& Hernández-Santamaría, P. O. (2017). La educación superior en Colombia: situación actual y análisis de eficiencia. Revista Desarrollo y Sociedad, 78, 59-111. https://doi.org/10.13043/DYS.78.2

Montes, J. A., Ayala, I. \& Atencio, D. F. (2005). Preparación para exámenes y aprendizaje autorregulado con estudiantes universitarios. Pensamiento Psicológico, 1(6), 57-71. Extraído de http://revistas.javerianacali.edu.co/index.php/pensamientopsicologico/article/view/20/57

Navea-Martín, A. \& Suárez-Riveiro, J. M. (2017). Estudio sobre la utilización de estrategias de automotivación en estudiantes universitarios. Psicología Educativa, 23, 115-121. https://doi.org/10.1016/j.pse.2016.08.001

Netemeyer, R. G., Bearden, W. O. \& Sharma, S. (2003). Scaling procedures: Issues and appliccations. Thousand Oaks, CA: SAGE.

Paulino, P. \& Lopes da Silva, A. (2011). Knowing how to learn and how to teach motivation: Contributions from self-regulation of motivation to more effective learning. Procedia-Social and Behavioral Sciences, 29, 656-662. https://doi.org/10.1016/j.sbspro.2011.11.289

Paulino, P., Sá, I. \& Lopes da Silva, A. (2015a). Autorregulacão da motivacão: Crenças e estratégias de alunos portugueses do $7^{\circ}$ ao $9^{\circ}$ ano de escolaridade [Autorregulación de la motivación: creencias y estrategias de alumnos portugueses en séptimo y noveno grado de escolaridad]. Psicologia: Reflexão e Crítica, 28, 574-582. https://doi.org/10.1590/1678-7153.201528316

Paulino, P., Sá, I. \& Lopes da Silva, A. (2015b). Crenças e estratégias da motivação na aprendizagem: Desenvolvimento de uma escala [Creencias y estrategias de motivación y aprendizaje: desarrollo de una escala]. Psychologica, 58(1), 65-87. https://doi.org/10.14195/1647-8606_58-1_4

Pett, M. A., Lackey, N. R. \& Sullivan, J. J. (2003). Making sense of factor analysis: The use of factor analysis for instrument development in health care research. Thousand Oaks, CA: SAGE. https://doi.org/10.4135/9781412984898

Pintrich, P. R., Smith, D. A. F., Garcia, T. \& McKeachie, W. J. (1993). Reliability and predictive validity of the Motivated Strategies for Learning Questionnaire (MSLQ). Educational and Psychological Measurement, 53, 801-813. https://doi.org/10.1177/0013164493053003024

Purdie, N. \& Hattie, J. (1996). Cultural differences in the use of strategies for self-regulated learning. American Educational Research Journal, 33, 845-871. https://doi.org/10.3102/00028312033004845

Rinaudo, M. C., Chiecher, A. \& Donolo, D. (2003). Motivación y uso de estrategias en estudiantes universitarios. Su evaluación a partir del Motivated Strategies Learning Questionnaire. Anales de Psicología, 19, 107-119. Extraído de https://www.redalyc.org/articulo.oa?id=16701911

Ryan, R. M. \& Deci, E. L. (2000). Intrinsic and extrinsic motivations: Classic definitions and new directions. Contemporary Educational Psychology, 25, 54-67. https://doi.org/10.1006/ceps.1999.1020

Sabogal Tinoco, L. F., Barraza Heras, E., Hernández Castellar, A. \& Zapata, L. (2011). Validación del Cuestionario de Motivación y Estrategias de Aprendizaje Forma Corta -MSQL SF, en estudiantes universitarios de una institución pública-Santa Marta. Psicogente, 14(25), 36-50. Extraído de http://revistas.unisimon.edu.co/index.php/psicogente/article/view/1855

Sansone, C., Fraughton, T., Zachary, J. L., Butner, J. \& Heiner, C. (2011). Self-regulation of motivation when learning online: The importance of who, why and how. Educational Technology and Research Development, 59, 199-212. https://doi.org/10.1007/s11423-011-9193-6

Sansone, C, Weir, C, Harpster, L. \& Morgan, C. (1992). Once a boring task always a boring task? Interest as a self-regulatory mechanism. Journal of Personality and Social Psychology, 63, 379-390. https://doi.org/10.1037/0022-3514.63.3.379

Schreiber, J. B., Nora, A., Stage, F. K., Barlow, E. A. \& King, J. (2006). Reporting structural equation modeling and confirmatory factor analysis results: A review. The Journal of Educational Research, 99, 323-337. https://doi.org/10.3200/JOER.99.6.323-338

Schwinger, M., Steinmayr, R. \& Spinath, B. (2009). How do motivational regulation strategies affect achievement: Mediated by effort management and moderated by intelligence. Learning and Individual Differences, 19, 621-627. https://doi.org/10.1016/j.lindif.2009.08.006

Schwinger, M., Steinmayr, R. \& Spinath, B. (2012). Not all roads lead to Rome - Comparing different types of motivational regulation profiles. Learning and Individual Differences, 22, 269-279. https://doi.org/10.1016/j.lindif.2011.12.006

Schwinger, M. \& Stiensmeier-Pelster, J. (2012). Effects of motivational regulation on effort and achievement: A mediation model. International Journal of Educational Research, 56, 35-47. https://doi.org/10.1016/j.ijer.2012.07.005

Schwinger, M., von der Laden, T. \& Spinath, B. (2007). Strategien zur Motivations regulation und ihre Erfassung [Estrategias para la regulación de la motivación y su medición]. Zeitschrift für Entwicklungspsychologie und Pädagogische Psychologie, 39, 57-69. https://doi.org/10.1026/0049-8637.39.2.57 
Suárez, J. M. \& Fernández, A. P. (2011). Evaluación de estrategias de autorregulación afectivo-motivacional de los estudiantes: las EEMA-VS. Anales de Psicología, 27, 369-380. Extraído de https://www.redalyc.org/html/167/16720051013/

Valencia Serrano, M., Duarte Soto, J. \& Caicedo Tamayo, A. M. (2013). Aprendizaje autorregulado, metas académicas y rendimiento en evaluaciones de estudiantes universitarios. Pensamiento Psicológico, 11(2), 53-70. Extraído de http://www.scielo.org.co/pdf/pepsi/v11n2/v11n2a04.pdf

Velicer, W. F. (1976). Determining the number of components from the matrix of partial correlations. Psychometrika, 41, 321-327. https://doi.org/10.1007/BF02293557

Wolters, C. A. (1998). Self-regulated learning and college students' regulation of motivation. Journal of Educational Psychology, 90, 224235. https://doi.org/10.1037/0022-0663.90.2.224

Wolters, C. A. (1999). The relation between high school students' motivational regulation and their use of learning strategies, effort, and classroom performance. Learning and Individual Differences, 11, 281-299. https://doi.org/10.1016/S1041-6080(99)80004-1

Wolters, C. A. (2003). Regulation of motivation: Evaluating an underemphasized aspect of self-regulated learning. Educational Psychologist, 38, 189-205. https://doi.org/10.1207/S15326985EP3804_1

Wolters, C. A. (2011). Regulation of motivation: Contextual and social aspects. Teachers College Record, 113(2), 265-283. Extraído de https://eric.ed.gov/?id=EJ927078

Wolters, C. A. \& Benzon, M. B. (2013). Assessing and predicting college students' use of strategies for the self-regulation of motivation. The Journal of Experimental Education, 81, 199-221. https://doi.org/10.1080/00220973.2012.699901

Wolters, C. A., Benzon, M. B. \& Arroyo-Giner, C. (2011). Assessing strategies for the self-regulation of motivation. En B. J. Zimmerman \& D. H. Shunck (Eds.), Handbook of self-regulation of learning and performance (pp. 298-312). New York, NY: Routledge.

Wolters, C. A. \& Rosenthal, H. (2000). The relation between students' motivational beliefs and their use of motivational regulation strategies. International Journal of Educational Research, 33, 801-820. https://doi.org/10.1016/S0883-0355(00)00051-3

Zimmerman, B. J. \& Martinez-Pons, M. (1986). Development of a structured interview for assessing student use of self-regulated learning strategies. American Educational Research Journal, 23, 614-628. https://doi.org/10.3102/00028312023004614

Zimmerman, B. J. \& Martinez-Pons, M. (1990). Student differences in self-regulated learning: Relating grade, sex, and giftedness to self-efficacy and strategy use. Journal of Educational Psychology, 82, 51-59. https://doi.org/ 10.1037/0022-0663.82.1.51

Fecha de recepción: Diciembre de 2016.

Fecha de aceptación: Octubre de 2018. 